\title{
The Application of Professor Du Yumao's Thought of "Back Against Heart” in Exogenous Fever
}

\author{
Jing Liu*, Wei Leng \\ Shaanxi University of Chinese Medicine, China.
}

How to cite this paper: Jing Liu, Wei Leng. (2021) The Application of Professor Du Yumao's Thought of “Back Against Heart” in Exogenous Fever. International Journal of Clinical and Experimental Medicine Research, 5(3), 349-352.

DOI: 10.26855/ijcemr.2021.07.018

Received: May 24, 2021

Accepted: June 17, 2021

Published: June 30, 2021

*Corresponding author: Jing Liu, Shaanxi University of Chinese Medicine, China.

Email: 937901265@qq.com

\begin{abstract}
Exogenous fever refers to a kind of disease with fever as the main symptom, which invades the human body by exogenous evil. In modern medicine, this kind of disease is mainly acute infectious disease, and includes the disease caused by bacteria or viruses with fever as the main symptom. Just as novel coronavirus, with fever, dry cough, fatigue as the main manifestations, a few patients with nasal congestion, runny nose, sore throat, myalgia and diarrhea and other symptoms. So exogenous fever is a common disease in clinic, and it has the characteristics of rapid onset and easy transmutation. Professor Du Yumao makes good use of the principle of concomitant treatment in the treatment of exogenous fever. In this paper, three aspects are discussed, namely, the combination of cold and warmth, the combination of supplementing and relieving, and the combination of elevating and elevating, so as to better illustrate the clinical application of the idea of concomitant in reverse, which is worthy of reference.
\end{abstract}

\section{Keywords}

Du Yumao, Back Together, Famous Doctor Experience, Exogenous Febrile

\section{Exogenous febrile}

Exogenous fever refers to a group of diseases with fever as the main symptom and various other syndrome types occurring in the process of disease [1] Chinese Medicine. Exogenous fever is relatively common in clinical practice, and some diseases have complex syndromes, such as COVID-19, which is currently under way. When the treatment effect of Western medicine alone is not good, Chinese medicine has accumulated rich experience in this field and has certain advantages. Professor Du, an expert on typhoid and rare diseases, believes that in the process of disease development and evolution, the total pathogenesis is the imbalance of Yin and Yang, and in the treatment of Yin and Yang should be adjusted to make them in a dynamic balance. Due to the mutual transformation between Yin and Yang, there are few symptoms of pure cold and pure heat in diseases, and they are often mixed with cold and heat. Therefore, on the basis of the materialist differentiation of Yin and Yang, the treatment concept of "back to back and reverse together" is proposed. The principle of its composition is that there is warmth in the qing dynasty, cool in the heat, complement in the attack, complement in the attack, and complement in the attack, scatter in the collection, collect in the scatter, fall in the rise, fall will be matched with rise, Yin from yangping, Yang according to Yin hiding, only to match nature every step. Professor Du Yumao academic thought of "the antinomy with" a preliminary study [2]. Professor Du believes that the pathogenesis of exogenous fever is nothing more than exogenous surface pathogenic factors or the occurrence and transformation of external pathogenic factors. The treatment concept of "reverse reverse combination" has a significant effect in the treatment of exogenous fever. The author summarized the characteristics of Dulao's "reverse reverse combination" method in the treatment of exogenous fever, 
and analyzed it as follows.

\section{With cold and warm, both inside and outside solutions}

Exogenous febrile, because of feeling cold evil appropriate XinWen nourish, feel the cool wind hot the evil should be symplectic nourish, for table evil unanswered, tired of heat into the table in the cold heat syndrome in a word, or fever, some physicians syndrome is not clear, still with wind heat treatment, usually choose cool simba nourish the dose of fructus forsythiae powder or sang ju Yin fire, often delay treatment. According to Yun Tieqiao, some doctors used exogenous fever too "light", so the third son was treated by mistake. However, the fourth son fell ill again, and the doctors added soy beans, gardenia, mulberry leaves and other light and cool products, which delayed the illness and aggravated the disease. In desperation, Yun tried ephedra soup to cure his son [3]. This also enlightens us that it is particularly important to distinguish the pathogenesis of exogenous fever. Professor Du put forward the method of cold and temperature and its usage here. This method was first derived from Inner Diameter [4]. Its advantage is that Xin Wen agent with a small amount of Xin Liang product, one can prevent Xin Wen agent is too dry, two for cold and heat mixed syndrome, can be flat cold and heat; Xin-liang agent with a small amount of xin-warm products, one can reduce heat at the same time can prevent the Xin-liang agent damage Yang, two can prevent the cool to restrain too much, Yu Fu external evil. The Hanwen prescription has broken the previous treatment principle of "cold is hot, hot is cold". Now the disease of exogenous fever is complex, and the combination of cold and warm mainly targets at the syndrome of superficial cold and mixed cold and heat, with remarkable clinical effect. The author summarized the application of cold temperature and its usage in exogenous fever in sorting out and studying the case of Dr. Du Lao. The summary is as follows.

According to Du Lao, exogenous fever occurs at first in the sun, and the sun is a disease, and the outer cold is the most common, while the inner heat changes, and the outer cold is unsolved, while the Yang qi is rather passive, and the internal heat changes [5]. Harmonious Battalion Guard; If the wind cold outside the bundle, Yang Yu Li heat syndrome, choose ephedra, cassia branch Xin Wen open Cou reason divergent table cold, gypsum Xin Han to Qingli heat in addition to trouble, Daqinglong soup cut; If the wind cold band surface, water drink to stop syndrome, choose Guizhi, Ephedra Jiebiao powder wind cold, dry ginger, Asarum warm drink, with peony acid cold Yin to prevent Yin consumption of blood, Xiao Qinglong soup cut; If the sun cold unsolved, the change in heat transfer becomes SAN Yang, notopterygium, radix puerariae, radix bupleuri Xie SanYang table of the cold, with cool plaster, radix scutellariae in heat, chai ge solution muscle soup cut, as the king of hotelling's gao-xu wang used six collections of scientific class party song note "said: this soup to qiang, ge, radix bupleuri with, and to assist such as gypsum, radix scutellariae, three Yang meridians is the rule table card, method of cold will turn heat" [6]. If the surface is cold and the heat is heavy, choose mint and Schizonep heidata to solve the evil in the table, mulberry bark, honeysuckle, scutellaria, and gesso to heat up, with wine rhubarb to heat down, double solution soup cut, this Fang Xin is warm and cool, to reach the double solution in the table. Du Lao proposed here that for those with high fever and cold aversion, it should be used with caution. First of all, it should be clear whether there is a symptom, "if there is a symptom of cold aversion, there is a symptom of cold aversion”. Secondly, it should be observed that changes in tongue coating and tongue quality should be observed [7].

If exogenous mistreatment damages the spleen and stomach, evil spirit invaginate, choose Coptis coptis, Scutellaria scutellaria bitter cold relief, dry ginger Pinellia Xin Wen disperses cold and stops vomiting, Pinellia Xiexin Decoction is cut; If evil into Shaoyang, Taiyin in the empty, water drink stop, choose Bupleurum, Scutellaria and Shaoyang evil heat, cassia, dry Jiang Xin Gan Yang, warm water drink, Chai Hugui branch dried ginger soup cut; Exogenous fever in the late stage of development, the formation of Yin and Yang deficiency, Yin and Yang are not aligned with the critical disease. If Yin in shengyang lattice, selected raw aconite root, dried ginger, scallion XinWen medicine back to Yang save inverse, Yin cold, but the original is still in, du old consider medicine, detained by Yin is cold, the heat can't display warm medicine medicinal properties, so add a XinHan agent as medicine led, treatise on febrile disease of China-Canada taste salty cold pig bile, du often substitute heartleaf houttuynia herb injection used for pig bile, XuanTong up and down, The clinical effect is remarkable. If exogenous fever in the late period of sudden vomit profits, Yang Qi decline, and Shenshen fainted delirium language, choose Si Ni plus ginseng soup and An Gong Niuhuang pills, Yang rescue Ni, wake up the mind and open the orifices.

\section{It can be used together to replenish and reduce diarrhea, and to promote positive and evil effects}

In exogenous fever, the good and evil are in conflict with each other, and the vital energy is not lost. If the Qi loss, 
evil overflowing, a virtual image, to Zhengzheng-based. Exogenous fever usually has rapid onset, rapid development and rapid transmission. The healthy qi is unable to fight evil, leading to both deficiency and reality. The traditional treatment principle of "reducing the deficiency and filling the deficiency" is not applicable here. If you choose the tonic agent, and fear the evil spirit to stay, evil spirit depression Fu. In exogenous fever, the good and evil are in conflict with each other, and the vital energy is not lost. If the Qi loss, evil overflowing, a virtual image, to Zhengzheng-based. Exogenous fever usually has rapid onset, rapid development and rapid transmission. The healthy qi is unable to fight evil, leading to both deficiency and reality. The traditional treatment principle of "reducing the deficiency and filling the deficiency" is not applicable here. If you choose the tonic agent, and fear the evil spirit to stay, evil spirit depression Fu.

Therefore, in the treatment of exogenous fever, it is necessary to distinguish whether there is deficiency of healthy qi, which deficiency of qi, blood, Yin and Yang belongs to, and to what extent [8]. If the external wind cold and dampness of the body Qi deficiency, there is a strong and hot hate cold, strong pain in the head, seize the pulse floating and heavy according to the weakness, it is appropriate to yiqi solution table, defeat sanhua cut; If the body Yang deficiency, exogenous cold surface syndrome, the main syndrome heat light cold heavy, seize the tongue and pulse characteristics, reconstituted Sanhua cut; If the severe cold, cold syndrome, fatigue force, ephedra asarum aconite soup cut; If the body Yin deficiency is exogenous wind heat, nourishing Yin solution, plus or minus soup is luxuriantly cut; Body blood deficiency exogenous cold, scallion white seven taste drink cut; If the persistent low fever, the main method is to replenish the healthy qi, dispel the pathogenic factors as a supplement, and to replenish the middle yiqi decoction and Yupingfeng powder, plus the antiseptic agent of sassu leaf, paraffin, Schizonep heidata, etc., which has a good clinical effect. According to the syndrome differentiation of six meridians, if evil into Shaoyang, small Chaihu decoction cut, reconciliation Shaoyang, Shuli Sanjiao; If evil into Shaoyang, and Yangming dry solid, large Chaihu decoction cut, reconciliation and through parallel, in order to solve the evil of Shaoyang and Yangming; If the sun disease caused by mistake under the spleen deficiency in the cold under the benefit and table syndrome fever, Guizhi Ginseng decoction cut, warm stop benefit; If the sun disease is mistreated, seriously injured heart Yang, cassia twig, licorice, dragon bone and oyster soup cut, warm tonifying deficiency heart Yang, calm palpitation; If the deficiency of Yin and Yang combined with cold surface light syndrome, ephedra aconite licorice decoction cut, warm the meridian to help Yang, light sweat solution. The most important part of the treatment method is to distinguish between deficiency and reality.

\section{Lifting phase cause, adjust smooth air machine}

Exogenous fever, if lost in Xuan, diarrhea, no sweat and other diseases; Loss in and health, stool constipation, cough and asthma and other diseases, pathogenesis attributed to the Qi machine adverse disorder, loss in Xuan health. Exogenous fever, if lost in Xuan, diarrhea, no sweat and other diseases; Loss in and health, stool constipation, cough and asthma and other diseases, pathogenesis attributed to the Qi machine adverse disorder, loss in Xuan health [9].

Bitter drop almond cough; If fever without sweat and dry stool, choose ephedra xuanfa jiebiao, rhubarb tongfu heat; If the external wind cold, Yingwei discord, choose cinnamon branch muscle sweat, peony Yin collect liquid and Yingqi, a loose and a convergence, to reconcile Yingwei; If the sun surface syndrome is not solved, heat into the large intestine, choose Pueraria root, take its natural flavor rise powder solution muscle fever, rise Yang stop diarrhea, Qinlian bitter cold down, Qingli heat stop profit; If the sun disease is mistreated and causes heat depression in the chest and diaphragm, gardenia fruit should be selected to clear the stagnation heat in the chest and diaphragm through the bitter cold, and tempeh should be used to relieve heat on the surface and reduce stomach qi. If the sun surface evil is not solved inside forced Yang Ming, large intestine to dereliction of duty, choose Kegen Tang solution table rise clear stop, Pinellia and stomach down reverse vomiting.

\section{Conclusion}

If the surface cold heat or cold heat mixed syndrome, choose cold temperature and usage, in order to achieve the surface and inside double solution; If the false and real mixed syndrome, the selection of reinforcing and reducing with the use of Zheng Da evil; If the air engine is lost in and down, the rise and fall phase is chosen to adjust the field air engine for the method. Professor Du makes good use of the idea of "back to back" to make prescription medicine, and adjusts the dynamic balance of Yin and Yang, which has good clinical effect. For example, the current COVID-19 epidemic is complicated, so we can draw lessons from Professor Du's treatment idea of exogenous fever and put forward this idea for the joint study, discussion and application of traditional Chinese medicine scholars. 


\section{References}

[1] Wang Xing, Du Xiaogang. Tracing the Origin of Exogenous Fever Theory in Traditional Chinese Medicine [J].

[2] Bu, F., Dong, Z. H. (2013). Chinese Journal of Traditional Chinese Medicine, 2013, 28(10): 2950-2952.

[3] Preliminary Study on Yun Tienniu Jiayu Qiao’s Academic Thoughts on Typhoid Fever [D]. Beijing University of Chinese Medicine, 2016.

[4] Liu Min, Zhang Yan. Analysis on the Rules of Combination of Cold and Temperature with Attack and Supplement in Treatise on Febrile Diseases and Its Clinical Application [J].

[5] Chen Jing-he. (2019). Introduction of experience in treating internal diseases with cold and warm combination [J]. New Traditional Chinese Medicine, 2019, 51(03): 297-299.

[6] Ding Shi Sanyang Toujie Tang. China Journal of Traditional Chinese Medicine, 2019, 34(09): 4120-4122.

[7] Hou, Y. J. (2002). Clinical observation of "Shuangjie Decoction" in the treatment of winter influenza [J]. Beijing Traditional Chinese Medicine, 2002(04): 231-232.

[8] Journal of Beijing University of Chinese Medicine (Clinical Edition of Traditional Chinese Medicine), 2003(02): 10-11.

[9] An, S. F., Yang, Y. J. (2019). The significance of contrastive and complementary drugs in Treatise on Typhoid and Meterious Diseases [J]. Chin Med Tribune, 2019, 34(02): 7-8. 